\title{
Upregulation of pro-inflammatory cytokines in the intercostal muscles of COPD patients
}

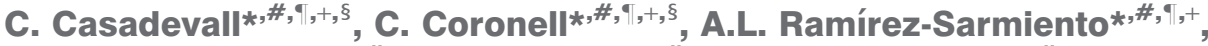

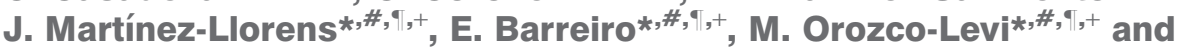 \\ J. Gea*,\#, ${ }^{*},+$ on behalf of the ENIGMA in COPD group
}

ABSTRACT: Muscle dysfunction is a characteristic feature of chronic obstructive pulmonary disease (COPD). Recent studies suggest that cytokines may operate as local regulators of both muscle function and regeneration. The aim of the present study was to characterise the expression of different cytokines in the external intercostal muscle of COPD.

Muscle biopsies were obtained from 25 stable COPD patients and eight healthy controls. Local

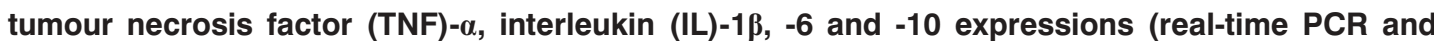
ELISA), sarcolemmal damage (immunohistochemistry), and the transcript levels of CD18 were assessed.

Muscle TNF- $\alpha$ and IL- 6 transcripts were significantly higher in COPD patients compared with controls, and IL-1ß and sarcolemmal damage showed a strong tendency in the same direction. Similar results were observed at protein level. The CD18 panleukocyte marker was similar in COPD and controls. Respiratory muscle function was impaired in COPD patients and it correlated to both the severity of lung function impairment and TNF- $\alpha$ muscle expression.

Chronic obstructive pulmonary disease is associated with the upregulation of pro-inflammatory cytokines in the intercostal muscles. This phenomenon might be involved in respiratory muscle dysfunction.

KEYWORDS: Chronic lung disease, interleukin-1, interleukin-6, muscle function, respiratory muscle, tumour necrosis factor- $\alpha$

hronic obstructive pulmonary disease (COPD) is characterised by non-fully reversible airflow obstruction. It is, however, a heterogeneous syndrome which, in addition to the classical involvement of lung parenchyma and airways, appears to be associated with a number of systemic manifestations. These include nutritional abnormalities, reduction of lean mass and muscle dysfunction. Central to this is the role that respiratory muscles play, since they represent the active component of the ventilatory pump. In COPD, respiratory muscles have to cope with increased workloads, mechanical disadvantages related to hyperinflation and, as recently demonstrated, an intrinsic reduction in their contractile properties [1, 2]. All these factors contribute to the impairment of the respiratory muscle capacity detected in these patients [2]. Despite a general consensus regarding this issue and its clinical consequences, little is known about the underlying pathogenic mechanisms that lead to impairment of the

For editorial comments see page 605 . intrinsic contractile properties of respiratory muscles. A growing amount of data, however, supports the involvement of cytokines and oxidative stress in the pathogenesis of muscle weakness in COPD [3]. Both loss of muscle mass and reduced strength have been attributed to increases in systemic cytokine levels, such as those of tumour necrosis factor (TNF)- $\alpha$ and interleukin (IL)-1 $\beta$ and -6 . Nevertheless, the presence of differences in the severity of muscle weakness among different muscle groups in stable COPD patients [4] indicates that, in addition to systemic factors, the process is probably regulated by intrinsic factors of the muscles themselves.

Recent studies indicate that skeletal muscles are capable of synthesising a variety of cytokines [5, 6], and that these cytokines could act as endogenous mediators of muscle dysfunction and/or adaptation via their autocrine/paracrine effects [7]. In the light of all of this, it was hypothesised that the increased activity of respiratory muscles might induce the upregulation of local proinflammatory cytokines in COPD, and that the
AFFILIATIONS

*Muscle and Respiratory System Research Unit (URMAR), Respiratory Medicine Dept, Hospital del Mar

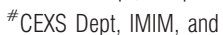

"Pompeu Fabra University, Barcelona, and

${ }^{+}$CIBER of Respiratory Diseases, ISCIII, Ministry of Health, Spain. ${ }^{\S} \mathrm{C}$. Casadevall and $\mathrm{C}$. Coronell share the position of first author.

CORRESPONDENCE

J. Gea

Muscle and Respiratory System Research Unit (URMAR) IMIM

Hospital del Mar

c/ Doctor Aiguader 88 E-08003 Barcelona

Spain

Fax: 34932213237

E-mail: jgea@imim.es

Received:

December 262005

Accepted after revision:

June 222007

\section{SUPPORT STATEMENT}

This study was funded in part by the following grants: QLRT-2000-00417 (PENAM project, EU), QLRT-200102285 (ENIGMA project, EU), SAF 2001-0426 (Plan Nacional I+D,

Spain) and RTIC C03/11 (Red RESPIRA-ISCIII, Spain)

STATEMENT OF INTEREST

None declared. 
latter may contribute to the pathogenesis of respiratory muscle weakness. Alternatively (or perhaps complementarily), the expression of these cytokines may play a role in the adaptive mechanisms that coexist with such functional muscle impairment. In both cases, local cytokine expression may also account for differences previously observed in the phenotype and function of different muscle groups in COPD [8-10]. Therefore, the purpose of the present study was to characterise the cytokine expression profile of a typical thoracic respiratory muscle (the external intercostal) in COPD patients.

\section{METHODS}

\section{Subjects}

In total, 25 clinically stable COPD patients and eight healthy subjects of similar age were included in the present study. While patients were consecutively recruited from the present authors' outpatient clinics, healthy control subjects were shared with a multinational study aimed at describing the effects of healthy ageing on muscle phenotype of elderly people across Europe (the PanEuropean Network for Ageing Muscle project). All COPD patients were ex-smokers $(47 \pm 13$ pack-yrs; mean period free of smoking $9 \mathrm{yrs})$, whereas none of the control subjects had ever been a smoker. In order to exclude sex-related effects, and on the basis of the COPD sex distribution in Spain, only male patients were included. Control Global Initiative for Chronic Obstructive Lung Diseases criteria were used to define COPD [11], whereas stability was defined as the absence of exacerbations during the 3 months before study entry. Subjects with chronic respiratory failure, metabolic diseases, cardiovascular problems, concomitant respiratory disorders, or treatments with drugs known to modify muscle structure or function were excluded. The study was designed according to the World Medical Association guidelines for research in humans [12] and was approved by the institutional ethics committee. Written informed consent was obtained from all individuals.

\section{Functional evaluations}

Lung and respiratory muscle function were assessed using conventional techniques. Briefly, forced spirometry with bronchodilator response (Datospir 92; Sibel, Barcelona, Spain), as well as intrathoracic gas volume, airway resistance and diffusing capacity of the lung for carbon monoxide (Masterlab; Jaeger, Würzburg, Germany) were measured in each individual. Blood samples were obtained from the radial artery of the nondominant arm and blood gas pressures were measured by conventional polarographic techniques (RapidLab 860; Bayer HealthCare, Newbury, UK). The strength of respiratory muscles was determined by measuring maximal respiratory pressures generated at the mouth during forced inspiratory (MIP) and expiratory efforts performed against an occluded airway from residual volume (RV) and total lung capacity (TLC), respectively. Reference values were those appropriate for a Mediterranean population [13-16]. Inspiratory muscle resistance was in turn assessed during two different threshold inspiratory tests [17]. The first was an incremental test, whereby patients and volunteers breathed against progressive loads ( $\sim 8 \mathrm{cmH}_{2} \mathrm{O}(0.78 \mathrm{kPa})$ every $\left.2 \mathrm{~min}\right)$ until maximal inspiratory sustainable threshold pressure $(P$ th,max $)$ was reached. This is considered a mixed outcome in terms of both endurance and strength components. In the second test, subjects breathed against a submaximal constant load $(80 \%$ of $P$ th,max) until exhaustion. The period that elapsed was defined as the endurance time, which is considered to be more specifically reflective of muscle resistance.

\section{Biopsies}

Samples from the external intercostal muscles were taken from the anterior axillary line at the sixth intercostal space, as previously described elsewhere [17].

\section{RNA isolation, reverse transcription and real-time PCR}

Total RNA was extracted from skeletal muscle using TRIzol reagent (Invitrogen Life Technologies, Carlsbad, CA, USA). Following quantification of total RNA, $1 \mu \mathrm{g}$ was reverse transcribed using oligo(dT)12-18 primer and superscript II reverse transcriptase (Invitrogen Life Technologies). cDNA synthesis was performed in a GeneAmp ${ }^{\circledR}$ PCR system 2400 (Perkin Elmer, Richmond, CA, USA), and an aliquot was used for real-time PCR amplification. This was performed with an ABI PRISM 7900HT Sequence Detector (Applied Biosystems, Foster City, CA, USA). Pre-developed TaqMan $®$ Assays were used to quantify transcripts for different cytokines (TNF- $\alpha$, IL$1 \beta,-6$ and -10), as well as for integrin CD18 (Assays-onDemand ${ }^{\mathrm{TM}}$ Gene Expression Products; Applied Biosystems), a global marker of leukocytes. $\beta$-Actin gene was used as the endogenous control (housekeeping). Probe context for the studied genes appear in table 1 . TaqMan ${ }^{\circledR}$ MGB probes were labelled at the $5^{\prime}$ end with the reporter dye molecule 6-carboxy-fluorescein and at the $3^{\prime}$ end with a nonfluorescent quencher.

Samples were always assayed in triplicate and the average value was taken (intra-assay variability coefficients $0.3-3.3 \%$ ). The PCR mixture was incubated for $2 \mathrm{~min}$ at $50^{\circ} \mathrm{C}$ for AmpErase ${ }^{\mathrm{TM}}$ uracil- $N$-glycosylase-mediated decontamination, followed by $10 \mathrm{~min}$ at $95^{\circ} \mathrm{C}$ to activate AmpliTaq Gold ${ }^{\mathrm{TM}} \mathrm{DNA}$ polymerase. Subsequently, a total of 50 cycles were performed; these consisted of a denaturation step for $15 \mathrm{~s}$ at $95^{\circ} \mathrm{C}$ and a combined annealing-extension step for $1 \mathrm{~min}$ at $60^{\circ} \mathrm{C}$. Data were analysed with the Sequence Detector software. The standardised target gene was compared with an external reference (i.e. a cDNA that was used in every assay). The relative copy number was calculated according to the comparative threshold cycle method [18].

\section{Protein level}

The cytokine contents were determined by ELISA performed using a commercial kit which included monoclonal antibodies against human TNF- $\alpha$, IL- $1 \beta,-6$ and -10 , according to the standard procedure recommended in the manufacturer's instructions (Amersham Pharmacia Biotech Limited, Amersham, UK). Briefly, $200 \mu \mathrm{L}$ of the sample were added to each well for a final dilution of 1:0.35. The microplates were then incubated for $3 \mathrm{~h}$ at room temperature and subsequently washed six times with $400 \mu \mathrm{L}$ of the appropriate buffer. Then, $200 \mu \mathrm{L}$ of the cytokine antibody were added to each well and incubated for $2 \mathrm{~h}$ at room temperature. Washes were repeated and $50 \mu \mathrm{L}$ of the substrate solution were added to each well and incubated for $1 \mathrm{~h}$ at room temperature. Immediately, $50 \mu \mathrm{L}$ of the amplifier solution were added and the mixture was again incubated for $30 \mathrm{~min}$ at room temperature. The process 
TABLE 1 Probes used for quantitative real-time PCR of the studied genes

\begin{tabular}{|c|c|c|c|}
\hline Genes & Assay identification & Nucleotide sequence $\left(5^{\prime}-3^{\prime}\right)$ & GenBank accession number \\
\hline TNF- $\alpha$ & Hs00174128_m1 & ATGTTGTAGCAAACCCTCAAGCTGA & NM_000594 \\
\hline IL-1 $\beta$ & Hs00174097_m1 & TATGGAGCAACAAGTGGTGTTCTCC & NM_000576 \\
\hline IL-10 & Hs00174086_m1 & CTACGGCGCTGTCATCGATTTCTTC & NM_000572 \\
\hline$\beta$-Actin $\#$ & Hs99999903_m1 & TCGCCTTTGCCGATCCGCCGCCCGT & NM_001101 \\
\hline
\end{tabular}

All TaqMan ${ }_{\circledast}$ minor groove binder probes were dual-labelled with a reporter dye (6-carboxy-fluorescein) at the $5^{\prime}$ end and a nonfluorescent quencher at the $3^{\prime}$ end. TNF: tumour necrosis factor; IL: interleukin. ${ }^{*}$ : housekeeping.

was stopped using a specific solution and the absorbance of each well was determined (Multiskan MS reader; Labsystems, Vantaa, Finland).

\section{Sarcolemmal damage}

The muscle section was stained with monoclonal antibodies against albumin (Research Diagnostics Inc, Flanders, NJ, USA) and the binding was visualised using the biotin-streptoaviodin-peroxidase technique (AB600, IC019; The Binding Site, Birmingham, UK), and developed with 3,3'-diaminobenzidine (D5337; Sigma, St Louis, MO, USA). Tissue was then observed under light microscopy and the image was digitised (Pixera Studio 1.2, Visual Communication System; Pixera Corporation, Los Gatos, CA, USA). Fibres showing immunopositivity for intrafibrillar albumin were identified and the percentage of positive cells was determined. In each case, $\geqslant 100$ fibres were evaluated by two independent and well-trained observers. These observers were blind with respect to sample identification. The edges of the section, as well as the areas with artefacts, were excluded from the analysis.

\section{Statistical analyses}

Data are expressed as mean $\pm \mathrm{SD}$. The normality of the distribution for each variable was assessed using the Kolmogorov-Smirnov test, and analysis of differences was performed using unpaired t-tests. The relationships between different variables were assessed using the Pearson's correlation coefficient. For the correlation analysis, both the COPD group and the (post hoc analysis) subgroup with a very severe disease (forced expiratory volume in one second (FEV1) $<30 \%$ predicted) were used. The analysis of the potential interactions between lung function, respiratory muscle function, sarcolemmal damage, the leukocyte marker and cytokine expressions was assessed using multiple regression analysis. Significance was accepted at $\mathrm{p}<0.05$.

\section{RESULTS}

\section{Comparison between COPD and controls}

General and anthropometric characteristics, as well as lung and muscle function data in both patients and control subjects, are indicated in table 2. Group mean values for age and body mass index were similar between COPD patients and control subjects. Whereas the COPD group showed a moderate-tosevere obstructive ventilatory defect, healthy controls presented normal pulmonary function. Inspiratory muscle strength and endurance were significantly lower in COPD with respect to the healthy subjects.

The levels of TNF- $\alpha$ and IL- 6 mRNAs found in external intercostal muscles from COPD patients were significantly higher than those observed in healthy controls $(p<0.001$ and $\mathrm{p}<0.01$, respectively; fig. 1a). IL-1 mRNA levels showed similar behaviour, although the difference did not reach statistical significance in this case $(p=0.09)$. Finally, no differences were observed for mRNA levels of the noninflammatory cytokine IL-10 between COPD and controls.

Protein levels of different pro-inflammatory cytokines showed similar behaviour to their homonymous mRNA. More pre-

\begin{tabular}{|c|c|c|}
\hline & Control & COPD \\
\hline Subjects $n$ & 8 & 25 \\
\hline Age yrs & $69 \pm 6$ & $66 \pm 5$ \\
\hline $\mathrm{BMI} \mathbf{k g} \cdot \mathrm{m}^{-2}$ & $26.0 \pm 2.5$ & $27.6 \pm 3.9$ \\
\hline \multicolumn{3}{|l|}{ Lung function } \\
\hline FEV $1 \%$ pred & $100 \pm 13$ & $34 \pm 12^{\star \star \star}$ \\
\hline $\mathrm{FEV}_{1} / \mathrm{FVC} \%$ & $72 \pm 3$ & $46 \pm 11^{\star * *}$ \\
\hline $\mathrm{RV} \%$ pred & $108 \pm 13$ & $176 \pm 39^{\star * *}$ \\
\hline TLC \% pred & $103 \pm 5$ & $105 \pm 17$ \\
\hline RV/TLC \% & $40 \pm 3$ & $63 \pm 8^{\star \star *}$ \\
\hline $\mathrm{Pa}_{1} \mathrm{O}_{2} \mathrm{mmHg}$ & $97 \pm 3$ & $65 \pm 3^{\star \star \star}$ \\
\hline \multicolumn{3}{|c|}{ Respiratory muscle function } \\
\hline MIP \% pred & $85 \pm 20$ & $65 \pm 22^{*}$ \\
\hline$P$ th, max $\mathrm{cmH}_{2} \mathrm{O}$ & $-66 \pm 22$ & $-40 \pm 19^{*}$ \\
\hline$t$ th80 $\min$ & $15.8 \pm 5.6$ & $10.7 \pm 5.8$ \\
\hline MEP \% pred & $93 \pm 23$ & $70 \pm 19 * \star$ \\
\hline
\end{tabular}

Data are presented as mean $\pm \mathrm{SD}$, unless otherwise stated. COPD: chronic obstructive pulmonary disease; BMl: body mass index; FEV1: forced expiratory volume in one second; \% pred: \% predicted; FVC: forced vital capacity; RV residual volume; TLC: total lung capacity; $\mathrm{Pa}_{\mathrm{a}} \mathrm{O}_{2}$ : arterial oxygen tension; MIP maximal inspiratory mouth pressure; $P$ th,max: maximum inspiratory sustainable pressure (obtained during progressive inspiratory threshold loading); th80: endurance time under constant submaximal inspiratory loads ( $80 \%$ of $P$ th, max) MEP: maximal expiratory mouth pressure. ${ }^{*}: \mathrm{p}<0.05$; ${ }^{* *}: \mathrm{p}<0.01$; ${ }^{* \star}$ : $\mathrm{p}<0.001$ all between control subjects and COPD patients. $1 \mathrm{mmHg}=0.133 \mathrm{kPa}$; $1 \mathrm{cmH}_{2} \mathrm{O}=0.098 \mathrm{kPa}$. 

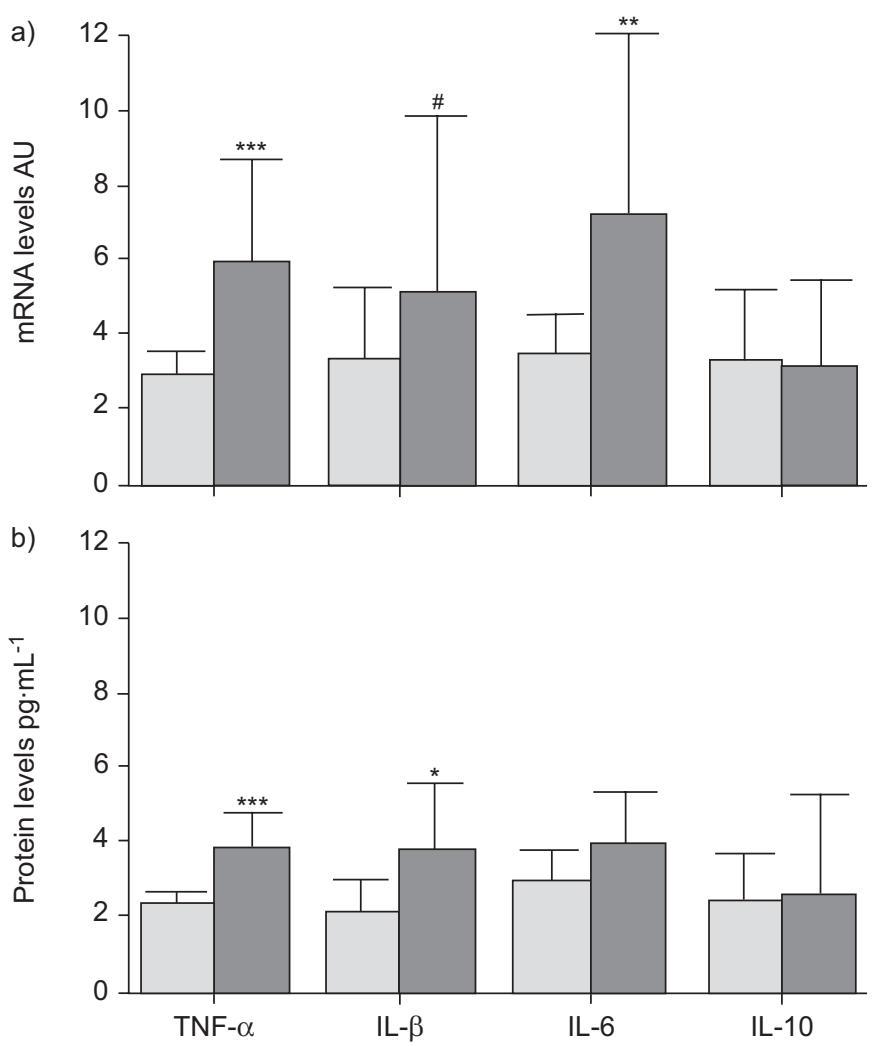

FIGURE 1. a) Relative cytokine mRNA levels and b) protein levels of the corresponding cytokines in external intercostal muscles of chronic obstructive pulmonary disease (COPD) patients ( $\square$ ) and control subjects ( $\square$ ). Error bars represent SD. AU: arbitrary units; TNF: tumour necrosis factor; IL: interleukin. ${ }^{*}: p=0.09 . *: p<0.05 ; * *: p<0.01 ; * * *: p<0.001$.

cisely, COPD patients showed significantly higher TNF- $\alpha$ and IL-1 $\beta$ protein levels than controls $(p<0.001$ and $p<0.05$, respectively), with IL-6 exhibiting a strong tendency in the same direction (fig. 1b). Sarcolemmal damage in turn showed a strong tendency to be higher in COPD patients ( $p=0.06$; fig. 2 ), whereas no significant differences were detected in the leukocyte marker (transcript for integrin- $\beta_{2}$ subunit CD18) [19].

\section{Correlations between lung and respiratory muscle functions}

The impairment in respiratory muscle function correlated with both the level of airway obstruction (FEV1 with either MIP $(\mathrm{r}=0.436, \mathrm{p}<0.05)$ or with $P$ th, $\max (\mathrm{r}=0.457, \mathrm{p}<0.01))$ and that of pulmonary hyperinflation (RV/TLC with MIP; $r=-0.479$, $\mathrm{p}<0.01)$.

\section{Intertranscript correlations}

The levels of TNF- $\alpha$ mRNA were positively correlated with the concentration of IL-1 $\beta$ mRNA $(r=0.567, p=0.001)$. This relationship was maintained unaltered when only COPD patients were considered $(r=0.565, \mathrm{p}<0.01)$. No significant associations were found between the leukocyte marker and cytokine levels.

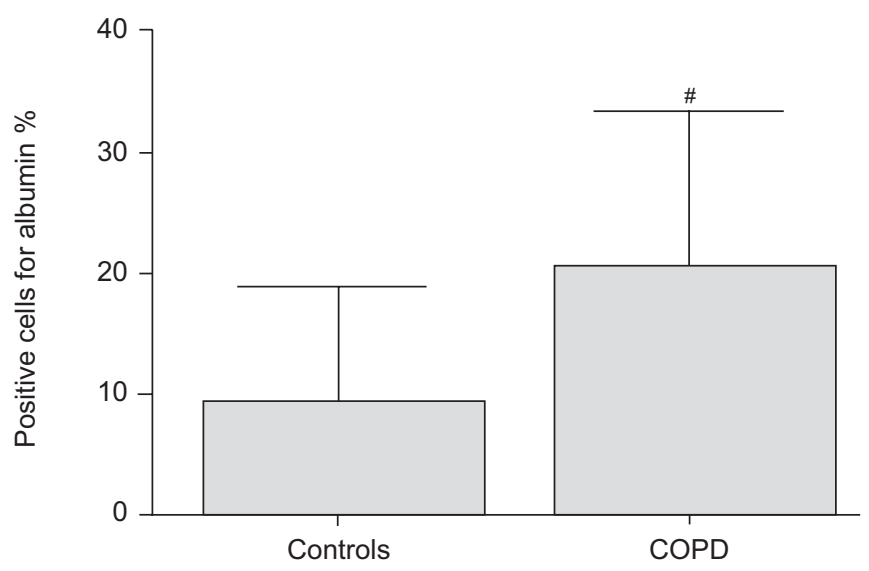

FIGURE 2. Sarcolemmal damage, assessed by the percentage of albuminpositive fibres, in the intercostal muscles of chronic obstructive pulmonary disease (COPD) patients and control subjects. ${ }^{*}: p=0.06$.

\section{Correlations between mRNA and protein levels}

These relationships oscillated moderately, with r-values ranging from 0.484 in the case of TNF- $\alpha(\mathrm{p}<0.01)$ to 0.583 in the case of IL-6 $(\mathrm{p}<0.001)$.

\section{Correlations between transcript levels and lung function}

No significant correlations were found between cytokine gene expressions and any of the nutritional and lung function parameters in COPD patients.

\section{Correlations between mRNA levels and respiratory muscle function}

TNF- $\alpha$ transcript showed an inverse relationship with $P$ th,max $(\mathrm{r}=-0.433, \mathrm{p}<0.05)$ in COPD patients. This relationship was even stronger $(\mathrm{r}=-0.666, \mathrm{p}<0.05)$ when only individuals with very severe disease (FEV1 $<30 \%$ ) were analysed (post hoc analysis); furthermore, the multiple regression analysis, which included lung volumes (as represented by RV/TLC) and the level of the TNF- $\alpha$ transcript, significantly increased the accuracy of the prediction for $P$ th,max in this population $(\mathrm{r}=0.743, \mathrm{p}<0.01)$.

Finally, sarcolemmal damage did not correlate with cytokine gene expression or with functional variables.

\section{DISCUSSION}

The main finding of the present study is the upregulation of TNF- $\alpha$ and IL-6 gene expressions observed in the external intercostal muscles of COPD patients, along with a similar tendency shown by IL-1 $\beta$. Protein levels followed a similar pattern. In addition, an inverse relationship was found between respiratory muscle function and the local muscle transcript of TNF- $\alpha$ in COPD patients.

Respiratory muscle dysfunction is commonly observed in COPD, and is believed to be the result of the combination of different deleterious factors [2-4]. Some are specific to these muscles, such as the changes occurring in the geometry of the thorax as a result of pulmonary hyperinflation. Others are general and, therefore, can be shared by respiratory and peripheral muscles: systemic inflammation, drugs and nutritional abnormalities, among others. However, molecular 
mechanisms occurring within different muscles are much less understood. Although the diaphragm is the main respiratory muscle at rest, other muscles, such as the external intercostals and parasternals, also participate actively in ventilation [20]. In addition, the intercostal muscles progressively play a more important role when there is an increase in ventilatory demands [21, 22].

Recent reports indicate that myocytes are capable of synthesising a variety of cytokines [5], which may act as regulators of skeletal muscle function and structure via an autocrine/ paracrine mechanism [23]. Furthermore, in a recent and significant paper, VASSILAKOPOULOS et al. [6] demonstrated that an increase in ventilatory loads induces the upregulation of different cytokines in the ventilatory muscles of rats. Based on these observations, it was hypothesised that local expression of pro-inflammatory cytokines is upregulated in the intercostal muscles of COPD patients, and that this upregulation may be contributing to the development of respiratory muscle weakness. Although the enhancement of the corresponding gene expressions has been confirmed by the present study, significant correlations with those variables reflecting muscle function were only found with the TNF- $\alpha$ transcript. TNF- $\alpha$ has long been associated with muscle catabolism in different diseases, including COPD [24]. In addition, it is commonly accepted that the loss of muscle mass is one of the main factors contributing to general muscle weakness in such patients [2]. Previous studies indicate that muscle function may be compromised by chronic elevation of circulating TNF- $\alpha$ via the development of oxidative stress [25], a phenomenon which has recently been reported within the respiratory muscles of COPD patients [26]. In addition to the action of systemic TNF- $\alpha$ on muscle, the present study also suggests that the local expression of this cytokine might have a role in respiratory muscle dysfunction, but probably in the context of a multifactorial scenario (as suggested by the multiple regression analysis). However, the present findings should be taken cautiously since they do not demonstrate a cause-effect relationship. Further studies should be designed to address this point.

In the present study, COPD patients also showed an upregulation of IL-6 gene expression in the intercostal muscle. Furthermore, this upregulation was related with the degree of airway obstruction. Recent studies demonstrated that IL-6 expression is induced by muscle contractions [27, 28]. Since inspiratory muscles of COPD patients face increased workloads, the present findings could be interpreted as an adaptive response to these higher contractile demands.

The third pro-inflammatory cytokine included in the study, IL-1, is considered a catabolic substance which, similarly to TNF- $\alpha$, has been shown to be able to induce contractile dysfunction through the presence of oxidative stress [29]. In the present study, the increase in IL- $1 \beta$ transcript bordered on statistical significance, but the corresponding protein was clearly augmented. In addition, a strong positive correlation was detected between the level of IL-1 mRNA and that of TNF$\alpha$. These findings suggest the existence of a synergistic action between both cytokines, which would provide a mechanism for increasing the inflammatory response within the respiratory muscles of COPD patients.
Local expression of TNF- $\alpha$ and other pro-inflammatory cytokines has been reported previously in human locomotor muscles $[5,30]$ and appears to be related in part to the level of muscle activity. However, the absence of significant correlations between lung function and local cytokine expressions in the COPD patients participating in the present study does not support a significant regulatory role for this factor, at least in the intercostal muscles.

Another factor that could partly account for changes in the expression of pro-inflammatory cytokines is fibre damage, since these substances play an important role in mediating muscle regeneration and healing [31]. In keeping with this, inspiratory loading has been reported to be associated with respiratory muscle damage [32] and subsequent remodelling [33]. In the present study, pro-inflammatory cytokine upregulation was associated with a marked tendency to show higher levels of sarcolemmal damage in muscles of COPD patients. Once again, although a cause-effect relationship cannot be inferred from the present data, the authors venture to speculate that a possible link exists between both findings. However, it is clear that further research will be necessary to identify the factors that lead to the overexpression of local cytokines observed in the intercostal muscles in the present study.

\section{Potential limitations of the study}

The possibility of obtaining valid specimens of the main respiratory muscle, the diaphragm, from human beings in vivo is very limited due to the need for very invasive surgical procedures and the potential bias derived from intrinsic comorbidity. In contrast, external intercostal muscles, whose main function is to assist inspiration when the diaphragm's function deteriorates or is insufficient to cope with the current workload, are more accessible [17]. In addition, the model used in the present study allows the possibility of a careful selection of both patients and controls, avoiding the problems resulting from comorbidity.

The second limitation of the present study is that it has been performed only in male patients. However, sex distribution of COPD is still markedly biased towards males in the present environment. The alternative of mixing both sexes in a common group with a net predominance of males was discarded, since the mechanisms conditioning and modulating muscle phenotype are very liable to be influenced by sex.

The third relative limitation of the present study is of a methodological nature, since the biological techniques employed are quantitative rather than topographic. In other words, they permit very precise quantification of cytokine levels, but their cellular source cannot be identified. Muscle biopsies, in addition to myocytes, may contain inflammatory and epithelial cells, which may also be a source of cytokines. However, the absence of differences for CD18 (a panleukocyte surface marker) transcript between COPD and controls suggests that inflammatory cell population was not increased in the former. This is in keeping with data published by GOSKER et al. [34], who were unable to find an increase in inflammatory cells in skeletal muscles of COPD. In addition, the lack of a relationship between the mRNA content for either TNF- $\alpha$ or IL- 6 and CD18 in the present study suggests that upregulation of these cytokines is not likely to be accounted for 
by the resident macrophages and infiltrated leukocytes in the intercostal muscles of COPD patients. Furthermore, previous studies using in situ hybridisation and immunohistochemical techniques have demonstrated that fibres are a major source of different pro-inflammatory cytokines in muscle [5].

In conclusion, the present study demonstrates that different pro-inflammatory cytokine genes are upregulated in the external intercostal muscles of chronic obstructive pulmonary disease patients. In addition, some evidence has been found to support the hypothesis that local expression of tumour necrosis factor- $\alpha$ might play a role in respiratory muscle dysfunction.

\section{ACKNOWLEDGEMENTS}

The authors would like to thank R. Marshall for his assistance with editing, and A. Roig and N. Soler (both Hospital del Mar, Barcelona, Spain) for their invaluable technical support.

\section{REFERENCES}

1 Levine S, Nguyen T, Kaiser LR, et al. Human diaphragm remodeling associated with chronic obstructive pulmonary disease: clinical implications. Am J Respir Crit Care Med 2003; 168: 706-713.

2 Skeletal muscle dysfunction in chronic obstructive pulmonary disease. A statement of the American Thoracic Society and European Respiratory Society. Am J Respir Crit Care Med 1999; 159: S1-S40.

3 Couillard A, Prefaut C. From muscle disuse to myopathy in COPD: potential contribution of oxidative stress. Eur Respir J 2005; 26: 703-719.

4 Gosselink R, Troosters T, Decramer M. Distribution of muscle weakness in patients with stable chronic obstructive pulmonary disease. J Cardiopulm Rehabil 2000; 20: 353-360.

5 Greiwe JS, Cheng B, Rubin DC, Yarasheski KE, Semenkovich CF. Resistance exercise decreases skeletal muscle tumor necrosis factor alpha in frail elderly humans. FASEB J 2001; 15: 475-482.

6 Vassilakopoulos T, Divangahi M, Rallis G, et al. Differential cytokine gene expression in the diaphragm in response to strenuous resistive breathing. Am J Respir Crit Care Med 2004; 170: 154-161.

7 Reid MB, Li Y-P. Cytokines and oxidative signalling in skeletal muscle. Acta Physiol Scand 2001; 171: 225-232.

8 Levine S, Kaiser L, Leferovich J, Tikunov B. Cellular adaptations in the diaphragm in chronic obstructive pulmonary disease. N Engl J Med 1997; 337: 1799-1806.

9 Whittom F, Jobin J, Simard PM, et al. Histochemical and morphological characteristics of the vastus lateralis muscle in patients with chronic obstructive pulmonary disease. Med Sci Sports Exerc 1998; 30: 1467-1474.

10 Gea JG, Pasto M, Carmona MA, Orozco-Levi M, Palomeque J, Broquetas J. Metabolic characteristics of the deltoid muscle in patients with chronic obstructive pulmonary disease. Eur Respir J 2001; 17: 939-945.

11 Global Initiative for Chronic Obstructive Lung Disease. Global Strategy for the Diagnosis, Management and Prevention of Chronic Obstructive Pulmonary Disease. www.goldcopd.com. Date last accessed: July 17, 2007.
12 World Medical Association General Assembly. World Medical Association Declaration of Helsinki: ethical principles for medical research involving human subjects. J Int Bioethique 2004; 15: 124-129.

13 Roca J, Sanchis J, Agusti-Vidal A, et al. Spirometric reference values from a Mediterranean population. Bull Eur Physiopathol Respir 1986; 22: 217-224.

14 Roca J, Burgos F, Barberà JA, et al. Prediction equations for plethysmographic lung volumes. Respir Med 1998; 92: 454-460.

15 Roca J, Rodriguez-Roisin R, Cobo E, Burgos F, Perez J, Clausen JL. Single-breath carbon monoxide diffusing capacity prediction equations from a Mediterranean population. Am Rev Respir Dis 1990; 141: 1026-1032.

16 Morales P, Sanchis J, Lamb PJ, Díez JL. [Maximum static respiratory pressures in adults. The reference values for a Mediterranean Caucasian population.] Arch Bronconeumol 1997; 33: 213-219.

17 Ramirez-Sarmiento A, Orozco-Levi M, Guell R, et al. Inspiratory muscle training in patients with chronic obstructive pulmonary disease: structural adaptation and physiologic outcomes. Am J Respir Crit Care Med 2002; 166: 1491-1497.

18 Livak K, Schmittgen TD. Analysis of relative gene expression data using real-time quantitative PCR and the $2^{-\Delta \Delta C}$ Tethod. Methods 2001; 25: 402-408.

19 Harris ES, McIntyre TM, Prescott SM, Zimmerman GA. The leukocyte integrins. J Biol Chem 2000; 275: 23409-23412.

20 DiMarco AF, Romaniuk JR, Supinski GS. Parasternal and external intercostal shortening during eupneic breathing. J Appl Physiol 1990; 69: 2222-2226.

21 De Troyer A. The electro-mechanical response of canine inspiratory intercostal muscles to increased resistance: the caudal rib-cage. J Physiol 1992; 451: 463-476.

22 DiMarco AF, Romaniuk JR, Supinski GS. Parasternal and external intercostal responses to various respiratory maneuvers. J Appl Physiol 1992; 73: 979-986.

23 Steensberg A, Keller C, Starkie RL, Osada T, Febbraio MA, Pedersen BK. IL- 6 and TNF- $\alpha$ expression in, and release from, contracting human skeletal muscle. Am J Physiol Endocrinol Metab 2002; 283: E1272-E1278.

24 Di Francia M, Barbier D, Mege JL, Orehek J. Tumor necrosis factor-alpha levels and weight loss in chronic obstructive pulmonary disease. Am J Respir Crit Care Med 1994; 150: 1453-1455.

25 Li X, Moody MR, Engel D, et al. Cardiac-specific overexpression of tumor necrosis factor- $\alpha$ causes oxidative stress and contractile dysfunction in mouse diaphragm. Circulation 2000; 102: 1690-1696.

26 Barreiro E, de la Puente B, Minguella J, et al. Oxidative stress and respiratory muscle dysfunction in severe chronic obstructive pulmonary disease. Am J Respir Crit Care Med 2005; 171: 1116-1124.

27 Pedersen BK, Akerstrom TC, Nielsen AR, Fisches CP. Role of myokines in exercise and metabolism. J Appl Physiol 2007; [Epub ahead of print PMID 17347387].

28 Banzet S, Koulmann N, Sanchez H, et al. Contractioninduced interleukin- 6 transcription in rat slow-type muscle is partly dependent on calcineurin activation. J Cell Physiol 2007; 210: 596-601. 
29 Cheng XS, Shimokawa H, Momii H, et al. Role of superoxide anion in the pathogenesis of cytokine-induced myocardial dysfunction in dogs in vivo. Cardiovasc Res 1999; 42: 651-659.

30 Rabinovich R, Figueras M, Ardite E, et al. Increased tumour necrosis factor- $\alpha$ plasma levels during moderate-intensity exercise in COPD patients. Eur Respir J 2003; 21: 789-794.

31 Kuru S, Inukai A, Kato T, Liang Y, Kimura S, Sobue G. Expression of tumor necrosis factor- $\alpha$ in regenerating muscle fibers in inflammatory and non-inflammatory myopathies. Acta Neuropathol (Berl) 2003; 105: 217-224.
32 Zhu E, Petrof BJ, Gea J, Comtois N, Grassino AE. Diaphragm muscle fiber injury after inspiratory resistive breathing. Am J Respir Crit Care Med 1997; 155: 1110-1116.

33 Gea J, Hamid Q, Czaika G, et al. Expression of myosin heavy-chain isoforms in the respiratory muscles following inspiratory resistive breathing. Am J Respir Crit Care Med 2000; 161: 1274-1278.

34 Gosker HR, Kubat B, Schaart G, van der Vusse GJ, Wouters EF, Schols AM. Myopathological features in skeletal muscle of patients with chronic obstructive pulmonary disease. Eur Respir J 2003; 22: 280-285. 